Horizons philosophiques

\title{
La dimension esthétique de l'existence humaine : pour une sociologie générale de l'esthétique
}

\section{Bruno Demers}

Volume 15, numéro 1, automne 2004

En quête du sujet

URI : https://id.erudit.org/iderudit/801279ar

DOI : https://doi.org/10.7202/801279ar

Aller au sommaire du numéro

Éditeur(s)

Collège Édouard-Montpetit

ISSN

1181-9227 (imprimé)

1920-2954 (numérique)

Découvrir la revue

Citer cet article

Demers, B. (2004). La dimension esthétique de l'existence humaine : pour une sociologie générale de l'esthétique. Horizons philosophiques, 15(1), 91-116.

https://doi.org/10.7202/801279ar d'utilisation que vous pouvez consulter en ligne.

https://apropos.erudit.org/fr/usagers/politique-dutilisation/ 


\section{La dimension esthétique de l'existence humaine : pour une sociologie générale de l'esthétique}

Qu'est-ce qu'être en tant qu'être humain? Qu'est-ce qu'être humainement? Voilà sans doute la question qui a le plus profondément traversé la réflexion philosophique depuis la pensée grecque et qui, encore aujourd'hui, réside au cœur du projet épistémologique des sciences humaines. Entre autres choses, "être humainement» signifie d'abord et avant tout entretenir corporellement, par l'intermédiaire des sens, un rapport sensible avec le monde au cours d'une existence qui se vit phénoménalement au travers des catégories du temps et de l'espace. Pour la sociologie, comme pour la philosophie, l'anthropologie, l'ethnologie et la paléontologie, comprendre ce que cela signifie d'être humain en ce monde, d'être un homo sapiens au sens fort du terme, implique donc nécessairement de prendre en considération cette donnée fondamentale de l'ontologie humaine qui sera appelée ici la dimension esthétique ${ }^{1}$ et de s'y attarder.

Or, la sociologie peut-elle investir cette dimension esthétique de l'existence humaine? Science de l'homme, la sociologie doit chercher à comprendre son objet sous ses moindres facettes et il est donc de son devoir de s'attaquer à tout ce qui entoure la sensibilité de l'être humain. Ce sera du moins l'objectif du présent article de montrer dans quelle mesure une approche sociologique peut contribuer à la compréhension de la dimension esthétique de l'existence de l'homme en société. Ses principales préoccupations seront d'amener le lecteur à cerner le fondement de cette dimension, à en déceler les caractéristiques et à comprendre ce qu'implique cette dimension au plan du rapport individu-société, cela tant au niveau des représentations que des pratiques. Pour mener à bien cette entreprise, une approche théorique et générale sera privilégiée, dans laquelle la nature de l'objet forcera le débordement du cadre strictement sociologique pour puiser dans d'autres matières telles que l'anthropologie, l'ethnologie et la philosophie. Pour cette raison, une démarche herméneutique sera implicitement présente tout au long de cet exposé : les matériaux choisis et utilisés en cours d'argumentation provenant d'auteurs, de 
paradigmes et donc de langages différents, il sera nécessaire de maintenir un exercice d'interprétation et de traduction des discours étudiés, afin de les intégrer dans la cohérence interne de ce travail.

Dans son premier volet, ce travail débutera par une présentation de la dimension esthétique du rapport au monde de l'homme. En survolant notamment un passage important des écrits de jeunesse de Karl Marx, cette première étape amènera dans le deuxième volet, à la manière de l'ethnographe, à faire un inventaire général des différentes manifestations de l'existence humaine qui sont spécifiquement esthétiques. Cela fait, le troisième volet tentera d'interpréter synthétiquement et analytiquement ces manifestations et de les mettre en lumière à l'aide des concepts généraux de style de vie et de normativité. C'est dans le quatrième volet qu'on tentera de situer la subjectivité humaine et l'activité artistique dans la dimension esthétique. Finalement, un retour sera fait sur les avenues parcourues au cours de ce travail sociologique et il sera possible de conclure en interrogeant la dimension esthétique des sociétés actuelles.

\section{L'ouverture anthropologique de la dimension esthétique}

Comme point de départ, un détour anthropologique est nécessaire afin de comprendre dans quelle mesure l'existence humaine comporte une dimension esthétique. À l'aide de ce détour, il faudra mettre successivement le doigt sur le moment constitutif et la nature d'une telle dimension.

\section{Le moment esthétique}

La question qui doit d'abord être posée est la suivante : à quel moment a-t-on commencé à entretenir au sein des sociétés un rapport esthétique avec le monde? L'être humain n'ayant de toute évidence jamais connu l'absence d'un tel rapport sensible au cours de l'évolution de l'espèce, certains diront qu'une telle question n'est que robinsonnade. Possible. II sera néanmoins maintenu qu'un tel moment peut analytiquement être souligné : un moment où en société l'homme a pris une distance avec l'ordre naturel et a cessé de se fondre avec celui-ci. II s'agit du moment de l'architecture, c'est-à-dire le moment où les hommes ont commencé à dominer et s'aliéner la nature, à l'architecturer. Dit autrement, il s'agit de l'instant où l'être humain a commencé à façonner un milieu à son image. L'architecture est ce moment où l'homme prend résidence dans le monde et l'«harmonise à ses fins propres 2 ", comme dans l'aménagement d'une 
clairière, d'une caverne, voire même dans le simple chant du primitif qui marche en forêt, et dont la foulée et l'écho constituent déjà une première architecturation du temps et de l'espace. Pour Michel Freitag, c'est en effet le moment de l'architecture qui marque l'ouverture d'une dimension esthétique : par l'architecture émerge «l'espace de la culture et de la norme au sein de la nature, espace autour duquel la nature se trouve alors par l'homme disposée et ordonnée comme monde, et dans lequel la société elle-même s'incarne de manière sensible ${ }^{3}$ ".

Moment esthétique fondateur, l'architecture rencontre ici l'art au sens grec, la technè, par laquelle la société sait circonscrire et se tailler un lieu d'asile dans le monde, un templum, théâtre sacré de son existence. Par cet acte esthétique originel, la société entreprend donc un premier contact sensible avec l'extérieur et entame sa domestication : c'est le passage d'un «espace subi à un espace construit 4». Elle prend en charge l'organisation spatio-temporelle des objets, de son milieu et de sa vie selon des formes et des rythmes qui lui sont propres. Bref, par ce moment architectural et architectonique, la nature se socialise, s'humanise et la société s'y matérialise en permettant à l'être humain d'accéder à l'espace de la culture et à un rapport esthétique au monde en tant que dimension constitutive de son existence sociale et sensible.

\section{La dimension esthétique de l'existence et les «lois de la beauté»}

Pour approfondir maintenant la nature de cette dimension esthétique qui s'ouvre à partir du moment de l'architecture, il faut la comprendre sous l'angle de la production de la vie matérielle. Sur la scène de ce monde humain sensible qui s'instaure désormais et à l'intérieur duquel les individus vont se mouvoir, il faut en effet se pencher sur la manière selon laquelle ils vont produire leur existence. L'analyse d'une section importante des Manuscrits de 1844 de Karl Marx divulguera à ce sujet de précieux renseignements :

Le mode d'activité vitale renferme tout le caractère d'une espèce, son caractère générique, et l'activité libre, consciente, est le caractère générique de l'homme (...) L'animal s'identifie directement avec son activité vitale. II ne se distingue pas d'elle. II est cette activité. L'homme fait de son activité vitale elle-même l'objet de sa volonté et de sa conscience (...) Certes, l'animal aussi produit. II se construit un nid, des habitations, comme l'abeille, le castor, la fourmi, etc. Mais il produit 
seulement ce dont il a immédiatement besoin pour lui et son petit; il produit d'une façon unilatérale, tandis que l'homme produit d'une façon universelle; il ne produit que sous l'empire du besoin immédiat, tandis que l'homme produit même libéré du besoin physique et ne produit vraiment que lorsqu'il en est libéré; l'animal ne se produit pas lui-même, tandis que l'homme reproduit toute la nature; le produit de l'animal fait directement partie de son corps physique, tandis que l'homme affronte librement son produit. L'animal ne façonne qu'à la mesure et selon les besoins de l'espèce, tandis que l'homme sait produire à la mesure de toute espèce et sait appliquer partout à l'objet sa nature inhérente; l'homme produit donc aussi d'après les lois de la beauté 5 .

Pour Marx, le propre de l'existence humaine est qu'elle repose sur une activité transcendante en regard des besoins de l'espèce, c'est-à-dire une activité qui ne connaît pas de limites et qui peut donc s'exercer dans un large univers de possibles. Contrairement à l'animal, l'homme ne se confond pas immédiatement avec son activité productrice et possède le pouvoir de faire de celle-ci une activité consciente et libre. L'animal peut certes produire et façonner son milieu, mais jamais à la manière de l'être humain. L'animal produit toujours "à la mesure et selon les besoins de l'espèce" : il suit la règle de l'espèce. À l'opposé - et c'est ce qu'on a vu avec le moment de l'architecture - l'homme a cette potentialité subjective d'،appliquer partout à l'objet sa nature inhérente", c'est-à-dire de se libérer de la nature et de produire son monde non pas selon la règle d'une espèce, mais selon une marge de manœuvre que l'animal ne connaît pas et, en conséquence, de pouvoir créer librement à la mesure de n'importe quelle espèce. De ce fait, Marx en conclut que l'homme façonne le monde d'après les "lois de la beauté", c'est-à-dire que tout le fruit de son travail serait régi par de telles "lois". Si l'on suit cette idée, puisque l'être humain se réverbère par son activité, non pas sur les seuls objets du travail, mais plus largement sur la totalité du monde, ce serait ici à la fois la nature, les produits du travail, les arts, les rapports sociaux et les individus eux-mêmes qui seraient façonnés selon les "lois de la beauté».

De plus, si l'on poursuit le raisonnement que Marx développe dans ses manuscrits de jeunesse, c'est dans cet univers entièrement esthétisé par l'homme selon lesdites «lois de la beauté» que s'ef- 
fectue la socialisation des sens de chaque individu - l'ouïe, la vue, la goût, l'odorat, le toucher : «le sens humain, l'humanité des sens, ne se forme que grâce à l'existence de leur objet, à la nature humanisée ${ }^{6}$ ". C'est la société dans laquelle les individus sont socialisés qui permet l'éducation et le déploiement de «la faculté subjective de sentir de l'homme 7 ". La société fait passer tout un chacun du statut d'animal humain à celui d'homme socialisé, et la sensibilité de chacun apparaît ainsi comme fonction de l'existence sensible de la société. C'est en ce sens que Marx affirme que chaque individu "se contemple (...) lui-même dans un monde qu'il a créé", car il s'y reconnaît dans un monde qui est son œuvre, l'œuvre perpétuelle de sa société qui ne cesse d'architecturer le monde selon les «lois de la beauté».

Si cette idée marxienne d'une production de l'existence et du monde humains selon les "lois de la beauté" est intéressante et permet de concevoir l'individu comme un être socialement esthétique et créateur, elle constitue cependant un sérieux problème quant à savoir ce que sont en vérité ces fameuses "lois". En effet, que signifieraient, du point de vue sociologique, ces dites "lois" de la "beauté»? Et d'où proviendraient-elles? Cela impliquerait-il l'existence de lois transcendantes et d'un «beau" absolu? Les réponses à ces questions, Marx les a malheureusement emportées dans sa tombe. Pour réussir à déchiffrer ce que Marx avait en tête, il faudra à présent continuer cette étude en allant explorer et questionner les manifestations esthétiques de la vie humaine et sociale. Cette visite de la galerie des manifestations esthétiques des sociétés permettra de faire après coup une relecture de cette idée de Marx, de voir à quel niveau d'analyse se situent ces "lois de la beauté", ainsi que de continuer l'investigation de la dimension esthétique de l'être humain. 


\section{Les manifestations esthétiques}

Voici atteint le point où il faut s'interroger concrètement à savoir à quels niveaux et sous quelles formes, au sein des sociétés, ces dernières se manifestent esthétiquement. Dans ce deuxième volet, cela consistera essentiellement à effectuer une radiographie générale de la dimension esthétique et d'en extraire les principaux spécimens de manifestations possibles. Pour ce faire, différents matériaux seront empruntés à Marcel Mauss et à André Leroi-Gourhan ${ }^{8}$. Sans nécessairement les voir dans l'ordre, cet inventaire fera la description des niveaux physiologique, technique, social et artistique des différentes manifestations esthétiques.

Le lecteur ne sera pas étonné d'entendre que l'intermédiaire par lequel une société manifeste son existence sensible est celui que procurent les sens de ses membres. Unique système de références spatio-temporelles, les sens forment le seul moyen par lequel l'homme se taille une place dans le monde. On pourrait nous objecter ici que la médiation du champ symbolique des représentations a, elle aussi, un grand rôle à jouer dans l'orientation des individus dans le monde. Certes, mais il ne faut pas perdre de vue qu'une telle médiation repose d'abord sur la codification de certains sens, comme le démontre l'exemple du langage verbal ayant l'ouïe comme fondement. Toute la dimension esthétique repose donc en fait sur ces constituants de la biologie humaine que sont les cinq sens, soient la gustation, l'olfaction, l'audition, la vision et le toucher, auxquels on doit à juste titre ajouter les sensations proprioceptives et cénesthésiques.

II faut d'emblée avoir en vue que toutes les manières qu'aura une société d'organiser la vie sensorielle des individus constitueront des manifestations esthétiques. II faut à ce sujet démentir l'idée préconçue selon laquelle l'esthétique ne renverrait qu'aux seules catégories de la vue et de l'ouïe, recoupant par-là les seuls arts plastiques et musicaux, puis renvoyant les autres sens et les autres manifestations esthétiques à la poubelle de l'existence sensible. L'ordre sensoriel humain ne se réduit pas qu'à ce que l'on voit et entend. Il doit être dit que les autres sens relèvent tout autant de la sensibilité humaine. Un mets goûté, un parfum porté ou un corps caressé sont des manifestations esthétiques au même titre qu'une sculpture observée ou qu'une mélodie entonnée. La dimension esthétique est la dimension de l'appréhension sensible du monde et il est épistémologiquement impardonnable de penser - parce que l'évolution biologique a favorisé chez l'homme une prépondérance de la vision et de l'audition 
- que le goûter, l'odorat et le toucher n'ont rien à voir avec l'inscription de l'homme dans l'ordre spatio-temporel du monde. Une telle croyance reviendrait à soutenir que l'individu victime de surdité et de cécité serait tout simplement privé de toute expérience esthétique. Beethoven n'eut-il pas une vie esthétique des plus riches qui soient malgré que son appréhension du rythme et du musical ne s'effectua à partir de sa surdité qu'à un niveau tactile? Ainsi, malgré la place prédominante occupée par la vue et l'ouïe dans l'ordre sensoriel et bien que cette prédominance ait pour conséquence qu'une grande partie des manifestations esthétiques et des arts renvoient à ces deux niveaux de la sensibilité, reste qu'il ne faut jamais oublier que les autres sens subissent eux aussi un ordonnancement au sein de la culture et participent donc également aux manifestations esthétiques des sociétés.

Cela dit, la gastronomie est un premier lieu où l'on peut commencer à explorer et à inventorier différentes manifestations esthétiques. En effet, toute la gastronomie des sociétés est constituée de phénomènes esthétiques dans lesquels le goûter, l'odorat, la vue et même le toucher buccal sont sollicités et organisés de façon précise. En plus de se jouer dans la décoration et la présentation visuelle des plats, l'esthétique alimentaire et culinaire se retrouve dans la manière dont chaque culture privilégie et fait "se succéder les effets de gustations dans un ordre fixe ${ }^{9}$ ”. De culture en culture, chaque société sait apprêter le capital alimentaire disponible en harmonisant les odeurs, le cuit et le cru, le chaud et le froid, l'épicé, le sucré, le salé, l'amer et l'acide selon des rythmes et des préférences relevant de stricte convention ethnique. Les mets sont en ce sens des manifestations esthétiques des sociétés. Les odeurs ont aussi leur place hors de la gastronomie. Leroi-Gourhan affirme que «l'olfaction conserve un rôle important dans les rapports (sociaux) ${ }^{10}$ \%. Nul ne contestera en effet toute l'importance prise dans chaque société par l'usage de parfums, de désodorisants ou l'esthétisation olfactive, non seulement du corps, mais aussi de certains lieux (encens d'églises, odeurs de centres d'achat, etc.)

Le corps est pour sa part un endroit capital où vienne se cristalliser une foule d'autres manifestations esthétiques, surtout quant à sa décoration. Mauss affirme même que "le premier objet décoré est le corps ${ }^{11}$ ». L'éventail des décorations corporelles est vaste et varie selon les cultures et les sous-groupes d'individus. II y a tout d'abord l'esthétisation directe du corps : désirabilité des tissus 
adipeux, peintures corporelles, tatouages, cicatrices, épilation, coiffures, chirurgies esthétiques et autres modifications s'appliquant à toutes les parties du corps. Par exemple, la circoncision, qui est chez les Juifs un acte religieux, "est avant tout une opération esthétique ${ }^{12}$ ». De même, la peinture des visages maoris, la taille des dents chez les Pygmées, l'excision du clitoris en Afrique et le culte du bronzage à l'américaine sont toutes des manifestations qui, en dehors de leurs caractères symboliques, sont fondamentalement esthétiques. Au même titre, l'ornementation et l'apparat constituent aussi des manifestations esthétiques : boucles d'oreilles, colliers, masques, bagues, bijoux sonores, etc. De plus, tout ce qui touche au vêtement - et il faut ici penser à l'industrie contemporaine de la mode - relève d'une stricte recherche esthétique et, comme le spécifie Mauss, "est avant tout parure plus que protection ${ }^{13}$ '. L'esthétique vestimentaire se manifeste à tous les interstices des sociétés et «donne l'exacte mesure de l'organisation ethnique et sociale ${ }^{14}$ » : chaque société a ses savoir-vivre vestimentaires; ses habillements féminins ou masculins; ses habits de travail, de fêtes, de nuits; ses parures politique, militaire, diplomatique, juridique, religieuse, sportive, etc.

L'esthétique se joue sur la décoration du corps, du vêtement, mais aussi sur toute autre forme d'embellissement ou d'enjolivure imaginable. II y a la décoration des objets usuels, du mobilier, des maisons, des jardins, des villes, et même des animaux domestiques. On touche déjà ici à la sculpture, la gravure, la peinture et l'architecture (beaucoup d'arts sont décoratifs). Pour toutes ces choses, Mauss parle de la présence d'une recherche esthétique dans laquelle «le motif esthétique vient se surajouter à un objet qui, par lui-même, n'a rien d'artistique, mais est destiné à des fins utilitaires ${ }^{15}$ ». L'esthétique vient s'additionner et se combiner avec l'utilité formelle des différents objets de la vie courante (armes, artefacts religieux, ustensiles, outils, moyens de transport, etc.) Tout objet est donc une manifestation esthétique, même celui marqué par "l'absence même de décoration ${ }^{16}$ ". Effectivement, la nudité du corps de telle tribu africaine ou la pure et stricte fonctionnalité de telle fourchette d'aujourd'hui restent dans leur minimalisme et leur simplicité un façonnement donné du monde sensible.

Par ailleurs, l'esthétique ne se restreint pas qu'aux objets. Comme le lecteur le pressentait peut-être déjà avec les manières d'être physiologiques et vestimentaires, plusieurs manifestations esthétiques se situent dans les comportements mêmes des individus. 
Les manières de se tenir pendant les repas, de se conduire en général, d'ordonnancer un rituel ou une cérémonie, de rythmer le pas ou le travail, de se représenter le propre et le sale, bref tous ces agirs qui balisent les sociétés sont eux aussi des esthétisations de l'existence. Gestes de communication, salutations, formules de politesse, forme du parler local et autres codifications du langage ne font pas exception. En résumé, toute la sphère de l'«étiquette est, en elle-même, quelque chose d'esthétique ${ }^{17}$ ". II faut conséquemment retenir comme manifestations esthétiques toutes les formes concrètes que prennent en société le bien-dire, le bien-agir, le savoir-jouir, le savoir-faire et le savoir-être.

Enfin, il faut répertorier dans les manifestations esthétiques tout ce qui s'apparente aux «arts", lesquels demeurent omniprésents dans toutes les sociétés et sont même indifférenciés de la vie réelle dans les sociétés dites primitives ${ }^{18}$. II faut mentionner les arts littéraires : prose, poésie, drame, tragédie, comédie, conte, etc. Viennent ensuite les arts musicaux de toute espèce, notamment la danse et le chant, qui, en sollicitant toute l'audition et la proprioception, contribuent à inscrire l'individu dans le temps et l'espace par des mouvements et des rythmes (pour Mauss l'homme est de fait «un animal rythmé ${ }^{19}$ »). Sur le plan de la vision, le domaine des arts plastiques vient boucler la boucle des manifestations esthétiques. Tant décoratifs, figuratifs qu'idéaux, les arts que sont le dessin, la peinture, la sculpture, la gravure, etc. sont présents dans toutes les sociétés. Ultimement, on peut rappeler et revenir à l'architecture qui est pour Mauss l'«art total', la mère des arts qui, suivant son moment esthétique originel, les commande tous en quelque sorte 20 . Toujours architecturation et modelage du domaine sensible, tout art est au fond architectural et tout artiste architecte du monde.

Bien qu'exhaustif cet inventaire reste succinct, superficiel et embrasse difficilement l'ensemble des manifestations esthétiques existantes. L'objectif n'étant ici que d'en donner un panorama global, il a été nécessaire d'escamoter certains pans considérables de la dimension esthétique des sociétés, dont notamment le ludique, l'érotique, le temporel et l'urbanistique. Que le lecteur sache seulement que les domaines des jeux et des sports, de la sexualité, de l'organisation sociale du temps et des rythmes de vie, ainsi que de la structuration à petite et grande échelles des espaces impliquent aux seins des sociétés l'existence de manifestations esthétiques tout aussi importantes que celles ici mentionnées. 
Malgré qu'il soit sommaire, cet inventaire n'en donne pas moins la mesure de la dimension esthétique des sociétés. L'architecturation du monde et la rythmicisation de l'homme dans le monde se manifestent à peu près partout : habitudes culinaires, objets usuels, décoration, habillement, bienséances, langue, arts de toutes espèces, etc. Bien qu'il fallût pour en faire la description la découper en niveaux et en catégories qui dans la pratique ne connaissent pas nécessairement de clivages réels, la dimension esthétique doit davantage être perçue comme un continuum de manifestations qui s'immiscent dans tous les pores de la vie culturelle de chaque société. D'ailleurs, pour Mauss, dans chaque chose réside en fait une part d'esthétique et c'est pourquoi l'étude d'un phénomène esthétique consiste essentiellement à étudier avant tout un côté de l'objet ou de l'activité en question'21. Tout phénomène social se présente en fait toujours comme un mélange inextricable de plusieurs éléments imbriqués, de sorte que la dimension esthétique renvoie uniquement à un aspect des types de manifestations sociales recensées ici - lesquelles peuvent toujours comporter, au-delà de leur dimension strictement esthétique, une ou plusieurs autres dimensions (économique, politique, juridique, éthique, morale, affective, religieuse, mystique, mythique, symbolique, narrative, technique, etc.).

Comme le lecteur l'a peut-être remarqué au fil des exemples rapportés jusqu'ici, on retrouve souvent une hybridation des dimensions techno-instrumentale et esthétique. Ancrées dans la matérialité, les choses esthétiques qui ont été répertoriées sont souvent reliées à un usage, une utilité (utilité technique, symbolique, religieuse, etc.), mais pour Mauss leur caractère proprement esthétique se distingue toujours par la présence de quelque chose de plus complexe que l'utilitéz2. Cela touche ici au rapport entre la forme, la fonction et la matière. Dans ce rapport, la forme, l'aspect esthétique de la manifestation, vient se surajouter à une fonction ou un usage social, aspect utile de la manifestation, tandis que la matière constitue de son côté le substrat et la contrainte technique de l'épanouissement esthétique de la forme (cela est particulièrement visible dans les arts où justement le technique rend possible et limite à la fois la manifestation esthétique). Le mets, le corps, la flèche, l'artefact religieux, la nage, le vêtement, la statue, le véhicule, l'habitation, la cérémonie et toute activité sociale ont chacun leur fonction culturellement déterminée, de même qu'ils ont chacun leur ancrage matériel spatio-temporel, et un extraordinaire éventail de modulations 
esthétiques de la forme reste possible dans et par la matière autour de la formule fonctionnelle ${ }^{23}$. La figure suivante, illustrant trois stylisations différentes d'un même objet, aidera probablement le lecteur à comprendre ce dont il est question.

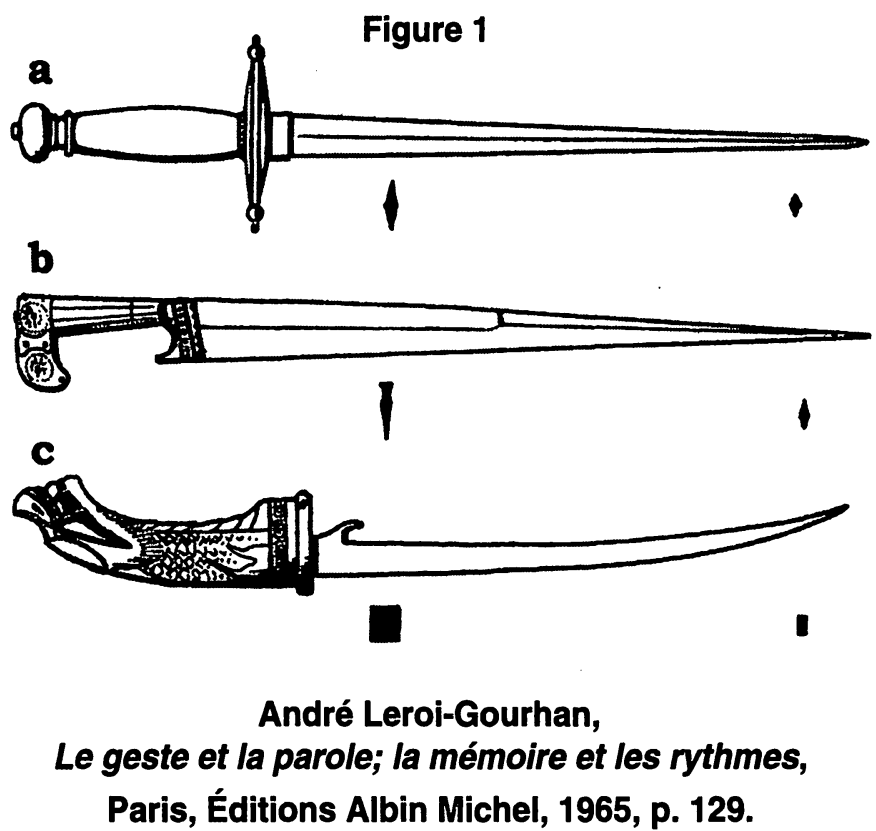

Cette figure représente trois dagues : a) européenne; b) iranienne; c) japonaise. Dans les trois cas, la fonction (traverser les armures légères) est la même et l'efficacité technique des trois modèles est équivalente. De son côté, la forme s'apparente à une enveloppe stylistique particulière à chacune des dagues venant auréoler leur formule fonctionnelle et technique. II y a donc dans la matière, autour d'une même fonction, un travail esthétique de la forme qui reste propre à chaque dague, à chaque culture.

Bref, comme on a tenté ici de l'illustrer, l'aspect esthétique émanant des choses apparaît en tout cas comme une façon particulière de les produire, une auréole, un surajout. Ainsi, dans ses diverses manifestations, la dimension esthétique semble relever d'un libre jeu stylistique et ethnique quant aux formes et aux rythmes qu'impose une société à son existence sensible. 


\section{Le style de vie comme normativité esthétique}

\section{Le style de vie}

L'inventaire général qui vient d'être fait se terminait en laissant sous-entendre le libre jeu stylistique à l'œuvre au cœur de la dimension esthétique des sociétés. En vérité, il est possible de proposer à présent une relecture de l'ensemble des manifestations esthétiques énumérées précédemment grâce à un concept sociologique synthétique, celui de style de vie. Ce concept n'est pas nouveau et on le retrouve chez plusieurs anthropologues et ethnographes, ainsi que chez des sociologues comme Roger Bastide24. Pour Bastide, toute société adopte une certaine "vision du monde" qui se laisse définir comme «un style de vie, une esthétisation du milieu physique et social25". À chaque époque, dans tout groupement humain et toute culture, cette vision s'exprime dans la manière propre d'une société d'organiser ses besoins, son milieu, puis d'y inscrire la vie et les comportements des individus. II s'agit du "goût» d'une société à un moment donné. À titre illustratif, Bastide reconnaît par exemple dans l'histoire de l'Occident le style de vie féodal centré sur l'esthétisation de l'amour et des rapports vassaliques; le style de vie monarchique avec son art baroque, ses règles d'étiquette et de politesse; etc. Le style apparaît comme le facteur esthétique déterminant de toute culture; la mesure que suivent les gens pour s'unir et qui donne la silhouette cohérente et l'allure commune que prennent l'être, le faire et l'agir des individus d'une société donnée, tels qu'ils peuvent prendre forme dans les divers types de manifestations préalablement survolées.

Les idées de Mauss et de Leroi-Gourhan vont dans le même sens que celles de Bastide. Le style de vie correspond chez Mauss "à l'ensemble du caractère esthétique dans lequel une société, à un moment donné, désire vivre 26 " et il entend par-là la totalité des éléments esthétiques d'une société (objets, instruments ou activités). Pour Mauss, le style de vie est tout spécialement «ce qui singularise une époque ou une société27". Leroi-Gourhan reconnaît également cette fonction singularisante du style de vie en y voyant la "tonalité culturelle ${ }^{28}$ " particulière dans laquelle baigne chaque groupe. Pour ce dernier, le style de vie peut se définir comme la manière qu'a une société de marquer les formes, les valeurs et les rythmes, et dans lesquelles la sensibilité individuelle est socialisée.

À résumer en des mots utilisés plus haut, le style de vie est la 
façon spécifique et unique qu'a toute société d'architecturer l'entièreté de son cadre d'existence sensible et d'y inscrire l'individu. II est facile à partir d'un tel concept de repenser les diverses manifestations esthétiques d'une société comme étant participantes d'un même style de vie. Sorte de méta-manifestation de son mode d'existence sensible, le style de vie devient ici l'«expression totale 29 " d'une société, à l'ombre de laquelle chaque petite manifestation particulière devient un épiphénomène tributaire de la tendance instituée et généralisée. Un simple objet, un geste, un vêtement, une mélodie, un motif architectural, un schéma urbanistique ou une figuration quelconque peuvent permettre de reconnaître une société donnée par ses seules apparences et appartenances stylistiques. Par exemple, tous sauront reconnaître en peinture ou en sculpture la stylisation du cheval japonais traditionnel de celle du cheval gothique. De même, le lecteur avait peut-être même reconnu d'emblée le style de chacun des trois poignards de la figure 1 comme appartenant respectivement aux cultures européenne, iranienne et japonaise.

Grâce à ce concept large et englobant, il devient aisé de relever différents styles de vie pour des cultures, des groupes ou des époques. On pourra dès lors distinguer les styles de vie de l'Égypte pharaonique, de la Chine ancienne, de la Rome impériale, de l'Athènes classique ou des défunts sculpteurs de l'île de Pâques; ceux des peuples maoris, esquimaux, canaques; de même que les esthétiques de la société industrielle, de la société de masse, des ethnies et des sous-groupes qui composent ces sociétés, ainsi de suite...

\section{Le langage du style de vie}

On vient de le voir, il est possible de se représenter l'ensemble des manifestations esthétiques d'une société comme relevant de son style de vie propre. Néanmoins, cela ne fait pas réellement progresser la compréhension sociologique de la dimension esthétique, si ce n'est que cela donne un bon outil descriptif pour appréhender cette dimension. C'est pourquoi il faut poursuivre et analyser l'essence du style de vie, afin de parvenir à percer le mystère rencontré chez Marx celui des "lois de la beauté" qui résideraient au fondement de toute manifestation esthétique et donc, à grande échelle, au fondement de tout style de vie.

Il fut mentionné plus haut que les manifestations dans lesquelles une société exprime son existence sensible ne sont jamais 
unidimensionnelles, mais constituent à tout coup un mélange inextricable et singulier d'éléments soit religieux, moraux, économiques, techniques, symboliques, esthétiques, etc. À cet égard, le champ symbolique du langage - qui dans les sociétés s'applique et se moule à la réalité vécue telle une matrice - est en intime relation avec les manifestations esthétiques d'un style de vie. Le grand ethnologue Maurice Leenhardt le décrit très bien en affirmant qu'au sein d'un collectif «le développement de l'expérience verbale et de l'expérience esthétique vont de pair 30 ». Ce qui est produit concrètement au niveau sensible, ce qui est manifesté esthétiquement, est aussi transposé et dit symboliquement dans la sphère du langage. Ainsi, si donc on analyse ce qui est dit symboliquement au niveau du langage, peut-être parviendra-t-on à comprendre ce qui est dit esthétiquement dans un style de vie.

C'est justement sur ce rapport entre langage et style de vie que Ludwig Wittgenstein s'est penché et dont, en vrai sociologue, il a fait l'analyse ${ }^{31}$. Wittgenstein s'intéresse à ce qui se trame derrière les jeux de langage entourant les manifestations esthétiques. II analyse les façons de vivre dans lesquelles les gens expriment des jugements de goût : ce morceau est "beau», ce portrait est "magnifique», ce pantalon est "trop court», ceci est "charmant», "que c'est merveilleux», etc. Naturellement, avec sa philosophie analytique, il ne cherche pas ce qu'est le "beau» dans l'absolu, ni en quoi le "beau» est plus ou moins différent du "sublime» ou du «joli». II prend ces événements comme des états de choses, comme des faits sociaux qu'il faut interpréter du point de vue de la logique formelle en ce qui a trait au sens qu'on doit leur attribuer.

Or, Wittgenstein affirme que ce ne sont pas les mots qui sont significatifs : "dans la vie réelle, lorsqu'on émet des jugements esthétiques, les adjectifs esthétiques tels que "beau", "magnifique", ne jouent pratiquement aucun rôle ${ }^{32}$ ". Accessoires, les mots peuvent être remplacés par des interjections («Ah !», «Hum !», «Oui !»...), des mimiques ou des gestes d'approbation ${ }^{33}$. Ce qui en fait importe au-delà des mots, affirme Wittgenstein, ce sont essentiellement les réactions esthétiques d'appréciation et de plaisir (ou de dégoût et de mécontentement) devant des circonstances concrètes que les individus ont été accoutumés à rechercher et apprécier (ou à éviter et déprécier). Ce qui se cache derrière ce comportement appréciatif en matière d'esthétique, c'est la rectitude, la conformité ou la nonconformité à des règles éthiques, car pour Wittgenstein il ne fait 
aucun doute qu' "éthique et esthétique sont une seule et même chose ${ }^{34}$ ", à savoir un ensemble de règles. II n'y a pas de réelle différence de signifiés entre les signifiants du «bien» et ceux du "beau", le "beau" et ses équivalents linguistiques ou réactionnels (gestes, mimiques, etc.) n'étant en vérité que des variantes du «bien», du "devoir-être", dans une certaine constellation de manifestations qu'on s'est arbitrairement habitué à percevoir comme faisant partie d'un pan de la vie sociale séparé du domaine de l'éthique, mais qui en réalité se rapporte tout autant à de telles normes.

Wittgenstein nous apprend que sous le schéma du «ceci est correct» ou "ceci ne l'est pas", les comportements positifs ou négatifs en matière de sensibilité ne sont pas autre chose que la satisfaction de normes éthiques prédéterminées qui s'expriment dans les manifestations esthétiques. Le raisonnement de Wittgenstein découle de la logique formelle : «il n'y a pas de sens à parler d'une route correcte (ou d'une manifestation esthétique telle) en dehors d'un tel but prédéterminé ${ }^{35}$ ". Un jugement de goût devant une manifestation esthétique quelconque ne peut exister que sur la base d'une norme sociale existant a priori et, en ce sens, l'esthétique en vogue dans le style de vie d'une société est l'expression d'un corpus de normes préétablies.

Conséquemment, le champ symbolique des jeux de langage et des réactions esthétiques qui ont occurrence devant la forme d'une maison, d'un acte, d'un tableau, d'une musique ou d'un simple pantalon, exprime toujours des prescriptions éthiques, des règles. Ainsi, ce qui derrière le symbolique se dit esthétiquement n'est donc autre chose que la norme. L'ensemble des manifestations esthétiques d'une époque, toutes les façons de vivre d'une culture ou d'un groupe donné, sont des préférences arbitraires qui renvoient à de tels comportements éthiques et normatifs. Le style de vie - ou, comme le dit Wittgenstein, "la façon correcte de vivre ${ }^{36}$ » - se ramène en somme à un ethos, une normativité.

\section{La normativité esthétique et les "lois de la beauté»}

Après analyse, le style de vie - ce "goût" du moment selon lequel des gens s'unissent suivant certaines règles - se rapporte à une certaine normativité cristallisée dans la culture de toute société et suivant laquelle la société produit son existence sensible, de même que la sensibilité de l'individu par voie de socialisation. Leroi-Gourhan résume bien la chose en affirmant qu'un style «constitue dans le 
temps et l'espace un code des émotions qui assure au sujet esthétique le plus clair de l'insertion affective dans la société37». En définitive, ce qui ressurgit de ce corpus de normes, de ces "lois" socialement instituées, ce sont les mystérieuses «lois de la beauté» de Karl Marx. Ces "lois" ne sont pas transcendantes, mais immanentes au social : elles sont littéralement l'incarnation de l'existence sensible de la société. Elles régissent l'être de la société dans sa manière de se manifester sur le plan esthétique et représentent ainsi son mode d'être spécifique dans le monde.

Toutefois, ces «lois», cette normativité, ne constituent pas une nécessité ontologique, mais forment toujours une contingence singulière de l'histoire des hommes en société. II y a là une dialectique fort subtile : les "lois de la beauté" déterminent tout autant la forme que prend le rapport sensible au monde des hommes que l'expérience sensible subjective des hommes participe, au fil du temps, à la construction et à l'institution de ces "lois", leurs "lois». L'être humain applique partout sa nature inhérente d'être générique, affirme Marx : partout il produit et applique ses "lois de la beauté" au fil de son architecturation du cosmos. L'humanisation du monde est aussi la légalisation progressive du monde. Marx aurait donc pu ajouter que les hommes ne produisent pas uniquement selon les "lois de la beauté», mais qu'ils produisent eux-mêmes ces «lois» par leurs participations subjectives individuelles à l'expérience sensible collective.

\section{La distance esthétique}

Les dernières avenues par lesquelles nous avons fait la relecture des «lois de la beauté» dont parle Marx viennent de faire ressortir, en ce qui concerne la question de la dimension esthétique des sociétés, l'importance de l'action des sujets humains, auxquels le concept sociologique de style de vie avait jusqu'ici laissé peu de place. Pris statiquement, par sa généralité, ce concept fait en effet abstraction de la subjectivité artistique et de toute expérience esthétique vraiment individuelle. Il est donc impératif que le concept de style de vie soit maintenant reconsidéré dynamiquement dans la dialectique individu-société et que soit identifié le rôle de l'expressivité individuelle en matière de jugement et d'art.

\section{Jugement et distance esthétiques}

D'où vient que l'individu puisse en société poser des jugements esthétiques, c'est-à-dire des jugements normatifs en "beau» ou en 
«bon» sur son propre style de vie ou même au-delà de celui-ci? La condition première de cette possibilité est qu'un style de vie ne peut pas - jusqu'à preuve du contraire - être parfaitement totalitaire. Comme Marcel Mauss et Pierre Clastres l'ont tous deux remarqué, même dans les sociétés primitives les plus restrictives où l'individu se fond dans le groupe, il y a toujours place à un minimum de libre expressivité individuelle, par exemple sous forme de licence poétique accordée à chacun lors des chants ${ }^{38}$. C'est un fait que les individus ne peuvent jamais être en parfaite adéquation avec l'objectivité du style de vie qu'ils endossent. Anthropologiquement, une distance esthétique existe toujours comme entre l'objectivité du style de vie d'une société et la sensibilité subjective des individus - sans quoi il s'agirait d'un système d'automates parfaitement objectifs, sans individualité, sans artiste, ni variante de style, ni changements au cours de la reproduction sociale. À ce sujet, Michel Freitag affirme que c'est justement dans cette dialectique que résident «la richesse (et) la condition existentielle fondamentale de toute existence humaine, sociale et historique ${ }^{40}$ ». Bien que l'individu s'imprègne du style de vie de son groupe, on ne peut omettre que ce style laisse place à un espace de subjectivation et d'autonomie. Le style de vie est mouvant, pluriel, toujours ancré dans les expériences esthétiques individuelles et se laisse enrichir de nouvelles normes par les individus. Au gré des expériences subjectives du monde, les styles de vie évoluent et leurs modulations sont aussi variées qu'il existe sur Terre de cultures, de générations successives d'individus dans ces cultures, de groupes, de distinctions sociales à l'intérieur des groupes et, à la limite, d'individus.

Partant, la possibilité du jugement de goût s'explique sociologiquement par ce qui vient d'être qualifié de distance esthétique inhérente à la relation entre la subjectivité de. l'individu et la normativité d'un style de vie, sans toutefois que le premier terme de la relation ne se laisse réduire au second. Le lecteur le pressentait probablement plus haut, la condition sine qua non du jugement de goût est la socialisation de l'être humain dans la normativité d'un style de vie. C'est l'immersion de l'individu dans des règles esthétiques qui lui permet de se prononcer sur les façons de vivre de sa société : "si je n'avais pas appris les règles, je ne serais pas en mesure de porter un jugement esthétique ${ }^{41}$ », nous dit Wittgenstein. En d'autres termes, il faut savoir les normes en vigueur, sans quoi il devient impossible de poser des jugements. Qu'on soit amateur, connaisseur ou expert de 
poésie anglaise, il faut premièrement, pour la juger, avoir appris les règles de la langue anglaise ! II en va de même pour toutes les autres manifestations esthétiques.

Ainsi, bien qu'il soit médiatisé par des normes extérieures, le jugement que pose l'individu n'en relève pas moins de ce dernier. Les jugements esthétiques demeurent toujours l'expression de sa volonté et cette "volonté est une prise de position à l'égard du monde (...) et de la vie ${ }^{42}$ ». Or, pour que la volonté s'exprime et prenne position de la sorte, il faut d'abord et avant tout qu'il y ait eu une appropriation subjective, par intériorisation sous forme d'habitus dans la psyché individuelle, de la normativité du monde vécu. Psychanalytiquement, en émergeant de la dialectique pulsions vs normes, le Moi ne se confond pas directement avec le Surmoi, gardant ainsi la possibilité d'une confrontation avec le principe de réalité. Sociologiquement, le processus social en matière d'esthétique corrobore le mécanisme psychique : en se construisant sur la dialectique expérience sensible subjective vs style de vie, le sujet intériorise en lui au cours du processus de socialisation la normativité esthétique de sa société sans toutefois que celle-ci ne le détermine complètement. Ce moment dialectique constitutif de l'individu est en puissance une distance esthétique. II s'agit d'une possibilité de distanciation qui, à rebours, permet à l'individu de se prononcer, par son discours ou sa pratique, sur le monde humain qui l'a fait naître.

\section{Arts et attitudes esthétiques}

Ainsi, bien que se manifestant collectivement dans ce que d'aucuns appellent des styles de vie, l'expérience esthétique du monde ne cesse jamais d'être individuelle et subjective. L'individu fait du nomos du monde humanisé son autonome : il tire du ou des styles dans lesquels il grandit le substrat normatif qu'il fait sien et à partir duquel il jugera les choses en "beau", en "bon", en "bien", etc. - les formules utilisées ne constituant pour leur part que des jeux de langage.

Subjectifs, les jugements sont des prises de position. Les jugements esthétiques n'ont pas comme référence des lois transcendantes, mais bien des «lois» immanentes au social à partir desquelles ils s'érigent et par rapport auxquelles ils peuvent opérer une. prise de distance. Leroi-Gourhan résume bien la chose en affirmant que l'individu tenant un discours esthétique le fait, "non dans l'absolu, mais dans la sécurité de l'esthétique du groupe et dans la liberté 
imaginaire de son choix ${ }^{43}$ ". Cela recoupe ici l'analyse que fait Kant de la faculté de juger : sur la base de l'universalité de l'expérience subjective, le sujet peut prétendre à un jugement universel, sans toutefois qu'il ne puisse jamais énoncer aucune règle absolue à laquelle il se réfère - la reconnaissance de la beauté par le jugement impliquant conséquemment chez Kant une "légalité sans loi44". Rendu en termes sociologiques, le diagnostic philosophique kantien exprime le fait de l'individu qui, aux prises avec l'inexistence d'une normalité en soi des formes sensibles, puise dans l'arbitraire et la contingence des "lois de la beauté" historiquement et culturellement accessibles pour tenir un discours normatif sur le monde - l'idée d'une "légalité sans loi» pouvant ainsi se relire sociologiquement comme celle d'une «normativité sans normalité».

Épanouie dans un discours, la faculté de juger devient le fait de l'individu qui peut enrichir par de nouvelles normes, par de nouvelles "lois de la beauté", la normativité esthétique de son style de vie en se prononçant sur le devoir-être des formes de l'existence sensible, que ce soit au niveau des manifestations en général ou au niveau des arts institués (pensons par exemple aux critiques d'arts ou à l'esthétique de Platon ou à d'autres théories esthétiques, lesquels ont tous en commun d'avoir des préoccupations normatives en matière d'esthétique ${ }^{45}$ ).

Cette faculté de juger, de tenir un discours normatif sur le "beau», est aussi en pratique pour le sujet la possibilité d'une action normative dans le monde. Par sa pratique, qu'il soit urbaniste, architecte, peintre, chanteur, écrivain, musicien, couturier, infographe ou même pâtissier à la limite, l'artiste peut poser des jugements de manière concrète, il peut prendre en objet son style de vie, enrichir ses formes, le façonner pour en célébrer ou en contester et en modifier les normes. Le propos de Jean Duvignaud est à ce sujet très instructif en ce qui concerne la position occupée par l'artiste au sein de la dimension esthétique des sociétés ${ }^{46}$. En effet, Duvignaud nous enseigne que la pratique de l'artiste - l'artiste étant ici entendu comme l'acteur dont l'activité est une pratique esthétique - se fait toujours en référence à un style de vie, c'est-à-dire à l'image que lui impose la société dans son architecturation du cosmos, et c'est «à cette image-là de la nature socialisée, affirme Duvignaud, que se réfère nécessairement l'artiste 47 ". Par son action, par l'incarnation de son individualité au travers de la forme et de la matière, l'artiste s'empare de cette image suivant le principe de distanciation énoncé 
pour le jugement de goût. L'artiste puise le substrat de son activité dans la normativité de la société et prend position par rapport à celle-ci. Les productions artistiques ne renvoient donc jamais directement à la nature ou la réalité elle-même, mais à "une nature doublement transposée; une première fois par la société, une seconde fois par un créateur 48 ». Cette transposition ne se fait cependant pas de façon unilatérale. Duvignaud informe qu'il existe plus d'une façon d'opérer cette transposition et que l'activité esthétique de l'artiste gravite entre deux pôles principaux.

À une extrémité, l'artiste peut reprendre à son compte le style de vie d'une société ou d'un groupe pour en exalter les manifestations esthétiques, les normes, les symboles 49 . Les arts auxquels on peut renvoyer ici sont ceux émanant directement de styles de vie : arts nationaux et arts de classe, arts d'élite et arts populaires, etc.. Pour des exemples plus spécifiques, on pensera aux arts traditionnels exaltant les thèmes religieux et l'ordre hiérarchique ou même au Pop Art, lequel reprenait par l'intermédiaire de médiums variés l'esthétique de la société de consommation en tentant de combler la distance entre l'art et la vie. On pensera également aux sociétés primitives dans lesquelles, comme il a été dit plus tôt, les arts font littéralement partie du style de vie. Dans de tels cas, la distanciation esthétique est à son plus bas.

À l'autre extrémité, Duvignaud ajoute que l'artiste «peut aussi luttef contre les codifications que lui impose une société 50 ». L'artiste cherche ici une distanciation esthétique plus négative ou, comme l'exprime bien Bastide, "une fuite hors du réel, du réel social autant que du réel physique, une évasion des contraintes qui pèsent sur (lui) 51 ». On pensera par exemple au romantisme qui, en évoquant par son lyrisme et son exotisme la nostalgie d'un ailleurs perdu, se mettait en porte-à-faux avec la société industrielle grandissante. Duvignaud insiste aussi en disant que ce deuxième pôle caractérise les arts faisant affront aux normes imposées par quelque société "sclérosée dans des systèmes de valeurs figés par des stratifications durcies ou par une société militaire 52 ». Sur ce point spécifique, on se rappellera le surréalisme et plus particulièrement l'automatisme québécois qui s'opposait aux normes strictes d'un régime encore traditionnel et constituait littéralement un Refus global de ce style de vie. Pour de tels cas, la distanciation esthétique est radicale.

Ces deux pôles sont évidemment deux types idéaux. Le positionnement artistique n'est jamais ou bien totalement pour, ou bien 
totalement contre la normativité esthétique d'une société. Placés au carrefour de toutes les forces du social, dans le drame de l'imbrication de toutes les dimensions de la société (éthique, religieuse, politique, etc.), les artistes naviguent entre ces deux positions idéales-typiques. À ce sujet, Duvignaud postule qu'on peut regrouper les positions qu'adoptent les artistes en diverses attitudes esthétiques. Selon les époques, on distinguera par exemple des attitudes allant, à partir des sociétés primitives, de la communion totale des artistes avec leur style de vie jusqu'à l'attitude d'artistes modernes s'opposant à l'ordre et aux valeurs établies, en passant par la quête de l'absolu chez certaines figures artistiques ou encore par les arts de l'illustration de la vie quotidienne ${ }^{53}$. Au cours de l'histoire de l'Occident, c'est à partir du début de la modernité que ces attitudes esthétiques ont graduellement fait place à une distanciation esthétique grandissante, visible tout particulièrement dans la doctrine moderne de l'art pour l'art.

\section{En guise de synthèse}

S'achève ici cet examen de ce qu'on a appelé la dimension esthétique. Décrivant d'abord schématiquement comment une telle dimension trouve sa place et son origine dans le moment architectural qui dégage de la nature un espace proprement humain et culturel, on a ensuite étudié chez Marx certaines considérations philosophiques sur le rapport esthétique au monde de l'être humain. Construisant alors notre problématique à partir de l'idée marxienne selon laquelle l'homme produit toute son existence selon les "lois de la beauté", cette existence a ensuite été explorée dans ses diverses manifestations esthétiques - manifestations qu'on retrouve à la fois dans le manger et le sentir, le savoir-être et le savoir-faire, les gestes, le parler, l'ornementation, les arts, etc. Ces différents types de manifestations esthétiques ont subséquemment été ré-encastrées au niveau culturel des sociétés en recourant au concept synthétique de style de vie. L'analyse des jeux de langage propres aux manifestations esthétiques a ensuite conduit à interpréter le style de vie comme la normativité qui définit en toute société le mode d'être qui lui est propre en matière d'existence sensible, et c'est dans cette normativité que notre travail herméneutique a permis de retrouver les fameuses "lois de la beauté" dont parle Karl Marx. Dans la dernière partie, la place de l'acteur, qui avait été jusque là volontairement mis de côté, a finalement été restituée à l'intérieur de cette normativité. À ce niveau, le propos s'est attaché à montrer que, par définition, le sujet humain 
n'est jamais entièrement déterminé par la société au niveau esthétique. Bien que l'individu soit ancré dans la normativité de sa culture et que celle-ci s'impose à lui de façon hétéronome, une prise de distance esthétique reste toujours une possibilité sous-jacente à sa nature de sujet humain sensible. Cette distance esthétique peut se réaliser tant au niveau théorique, sous formes de jugements et de discours, qu'au niveau pratique, par les arts, lesquels peuvent être rattachés à diverses attitudes esthétiques que l'on retrouve dans I'histoire des sociétés jusqu'à aujourd'hui.

Bien qu'ayant à coup sûr contribué d'un point de vue sociologique à éclaircir certaines choses concernant la question de l'esthétique, ce travail reste essentiellement théorique et ne prétend d'aucune manière avoir épuisé la richesse du problème. II s'est centré sur l'analyse de la dimension esthétique des sociétés en extrayant celle-ci de la multidimensionnalité du social et n'aura mis son emphase sur aucun matériau empirique en particulier. Cela dit, il reste néanmoins pertinent de conclure en posant un regard sur l'état actuel de la dimension esthétique de nos sociétés. À ce sujet, nul besoin n'est de décrire toute la diversité et l'abondance des manifestations, la multiplicité des goûts, des publics et des attitudes esthétiques, la profusion des arts, des lieux de diffusion, des médiums et des nouvelles pratiques esthétiques (graffitis, body piercing, expériences virtuelles, activités de cirque ou humoristiques, etc.), l'affluence des créations en tous genres, ainsi que la superposition des styles de vie qui y règnent et qui constituent le style de vie d'aujourd'hui. Qu'elles soient d'origine ethnique ou non, les manières, les préférences et les modes les plus variées (verbales, vestimentaires, corporelles, culinaires, artistiques ou autres), de même que toutes les danses, les musiques, les littératures et les architectures, sont dorénavant en permanent côtoiement dans une société enrichie par les formes et les rythmes de plus de deux mille ans d'histoire.

Maintenant que se sont diversifiés les styles de vie, la vie imaginaire et les créations, le propre du contexte actuel est de rendre possible la coexistence de tous les styles et de toutes les attitudes esthétiques, mais c'est également aujourd'hui que la norme en matière d'esthétique devient problématique. L'homme crée désormais selon toutes les normes esthétiques, selon des critères de beauté qu'il génère sans cesse lui-même. En ce sens, jamais il n'aura été plus vrai d'affirmer comme Marx que l'homme produit selon ses «lois de la beauté». Cependant, que dire devant cette réalité actuelle, si riche et 
plurielle soit-elle dans sa diversité et dans sa mémoire des styles de toute époque et de tout lieu? Que penser de cette dimension esthétique où la normativité des styles est devenue hétérogène et multicolore, et où jamais auparavant la notion même de "beau» n'a été aussi problématique?

Les avis divergent à ce sujet. Certains en font l'apologie en affirmant qu'il s'agit d'un «nouveau milieu, pour un sujet nouveau, pour des hommes plus libres et plus créateurs ${ }^{54}$ ». Dans une société ayant une normativité esthétique plus flexible et offrant plus de possibilités (techniques notamment), on pourrait en effet y voir un champ plus large pour la subjectivation, l'expression de soi et la réalisation effective du caractère générique de cet homme marxien, lequel pourrait enfin produire à la mesure de toutes les espèces et selon toutes les lois. $A$ contrario, loin d'y voir l'épanouissement des possibles de l'homme, les plus critiques y dénonceront le danger du "développement d'une "esthétique"» de l'expression arbitraire de soi (...) hors de toute participation culturelle synthétique ${ }^{55}$ » et de l'inscription de l'individu dans «une nouvelle condition d'assujettissement de la personne à une totalité sociétale en voie de devenir immédiatement objective sous la forme d'un système qui englobe et gère tout de manière pragmatique et opérationnelle ${ }^{56}$ '. S'il s'avérait que, dans leur parfait pluralisme, nos sociétés soient traversées par une expressivité esthétique individuelle à la carte, une évasion esthétique en vérité téléguidée, soumise aux industries culturelles, à la doxa conformiste de l'anticonformisme et au diktat de la consommation, on pourrait tout aussi bien penser que la fin d'une réelle normativité en matière d'esthétique concourrait au rétablissement d'une forme d'hétéronomie.

Devant cette divergence des opinions, il est possible d'imaginer que le contexte contemporain comporte un peu de ces deux tendances; la liberté acquise par l'évacuation des normes fixes évacuant aussi la possibilité pour l'acteur d'une action vraiment normative dans le monde, la ligne n'aurait alors jamais été aussi mince entre la plus grande liberté et la plus grande servitude. Si dans le meilleur des mondes la première tendance paraît la plus désirable, la seconde n'en reste pas moins effroyable. L'issue de la tendance dite postmoderne vers un système de l'immédiat 57 impliquerait un retour à une jungle objective surhumanisée, une déshumanisation du monde et la fermeture de la dimension esthétique telle que connue jusqu'ici. Du coup, cela pourrait signifier la clôture de la distance 
esthétique dont nous parlions plus tôt et donc la fin de l'art, de la sensibilité réellement subjective et de la beauté! Évidemment, nous n'en sommes pas encore arrivés à ce seuil. On peut de toute façon rassurer le lecteur en lui rappelant que, si l'expérience esthétique du monde tendait éventuellement à disparaître, il reste qu'elle pourrait revenir à la charge à tout moment, car si l'être humain cesse un jour d'être esthétique en acte, il ne cessera par contre jamais d'être en puissance un être esthétique et artistique.

\section{Bruno Demers \\ Sociologie,université du Québec à Montréal}

1. Dans un souci de rigueur méthodologique, il est nécessaire de préciser que le vocable "esthétique" sera utilisé dans cet article à titre de substantif des catégories kantiennes du temps et de l'espace, ainsi qu'à titre d'adjectif pour qualifier tout ce qui entretient un rapport avec ces catégories. C'est en ce dernier sens d'un rapport au temps et à l'espace par l'entremise des sens que nous parlerons respectivement pour l'homme de "sensibilité" et d' "existence sensible", soit une existence s'organisant au travers du temps et de l'espace de même qu'organisant le temps et l'espace. De plus, le terme "esthétique» entretiendra aussi une relation avec ce qu'on appelle le "jugement de goût", ainsi qu'avec la "beauté" ou le "beau», mais ce ne sont pas là des concepts que nous tiendrons d'emblée dans notre propos; notre propos aura plutôt affaire à donner progressivement une signification sociologique à chacune de ces expressions.

2. Michel Freitag, Architecture et société, Montréal, Éditions Saint-Martin, 1992, p.15.

3. Ibid., p. 17.

4. André Leroi-Gourhan, Le geste et la parole; la mémoire et les rythmes, volume II, Paris, Éditions Albin Michel, 1965, p. 140.

5. Karl Marx, Manuscrits de 1844, Paris, Éditions sociales, p. 62-64.

6. Ibid., p. 94.

7. Ibid., p. 93.

8. Nous nous référons à l'ethnographie de l'esthétique de Marcel Mauss : "Esthétique», chapitre 5 du Manuel d'ethnographie, Paris, Payot, 1967, p. 85-135; ainsi qu'à la paléontologie des symboles d'André Leroi-Gourhan : "Les symboles ethniques", Le geste et la parole, volume II, troisième partie, op. cit., p. 79-256.

9. André Leroi-Gourhan, op. cit., p. 110.

10. Ibid., p. 114.

11. Marcel Mauss, op. cit., p. 96.

12. Ibid., p. 98.

13. Ibid., p. 100.

14. André Leroi-Gourhan, op. cit., p. 194.

15. Marcel Mauss, "L'art et le mythe d'après M. Wundt", Revue philosophique de la France et de l'étranger, № 66, juillet à décembre 1908. 
La dimension esthétique de l'existence humaine :

pour une sociologie générale de l'esthétique

16. Marcel Mauss, «Esthétique», op. cit., p. 102.

17. Roger Bastide, Art et société, Montréal, L'Harmattan, 1997, p. 188.

18. Michel Freitag, "Notes pour une sociologie de l'art", in Sociologie de l'art, no 4, Presses de Belgique, Bruxelles, 1994, p. 45.

19. Marcel Mauss, "Esthétique», op. cit., p. 109.

20. Ibid., p. 108

21. Ibid., p. 85.

22. Ibid., p. 86.

23. Voir Leroi-Gourhan, op. cit., chapitre xii, p. 120-137.

24. Le concept de style de vie développé par Bastide (Art et société, Montréal, L'Harmattan, 1997) connaît également diverses variantes : Mauss parle tout simplement de "style"; Leroi-Gourhan préfère l'appellation "style ethnique"; Maurice Leenhardt utilise à certaines reprises l'expression "cadre de vie" (Do kamo, Paris, Gallimard, 1971). Notons également que Ludwig Wittgenstein, auquel nous nous référerons plus loin, utilise lui aussi le concept de «style de vie" (Leçons et conversations, Paris, Gallimard, 1992, p. 32). Dans tous les cas, les signifiants qu'emploient ces auteurs renvoient à peu de choses près au même signifié exposé ici.

25. Roger Bastide, op. cit., p. 190.

26. Marcel Mauss, op. cit., p. 103.

27. Ibid., p. 88.

28. André Leroi-Gourhan, op. cit., p. 90.

29. Ibid., p. 93.

30. Maurice Leenhardt, Do Kamo, Paris, Gallimard, 1971, p. 179.

31. Ludwig Wittgenstein, Leçons et conversations, Paris, Gallimard, 1992.

32. Ibid., p. 19.

33. Ibid., p. 21.

34. Ludwig Wittgenstein, Tractatus logico-philosophicus, aphorisme 6.421, Paris, Gallimard, 1992, p. 110.

35. Ludwig Wittgenstein, Leçons et conversations, op. cit. p. 147.

36. Ibid. p.144.

37. André Leroi-Gourhan, op. cit., p. 82.

38. Marcel Mauss, «Esthétique», op. cit., p. 133, ainsi que Pierre Clastres, La Société contre l'État, "L'arc et le panier», chapitre 5, Les Éditions de Minuit, Paris, 1974.

39. Cette formule est empruntée à Daniel Bell telle qu'on la retrouve dans Les contradictions culturelles du capitalisme, Paris, PUF, 1979, p. 125.

40. Michel Freitag, «Notes pour une sociologie de l'art», op. cit., p. 45.

41. Ludwig Wittgenstein, Leçons et conversations, op. cit., p. 23.

42. Ludwig Wittgenstein, Carnets, p.160, in Ibid., p. 165.

43. André Leroi-Gourhan, op. cit., p. 94.

44. Emmanuel Kant, Critique du jugement, 1790, in Encyclopaedia Universalis, loc. cit., p. 726. 
45. En effet, comme le dit Bastide, "les préoccupations normatives se sont inextricablement mêlées aux descriptions théoriques» dans les discours esthétiques (op. cit., p. 179). Souvent dans l'histoire de la pensée, des jugements sont venus colorer des discours esthétiques à prétention scientifique, les descriptions de fait étant donc en vérité des prescriptions morales.

46. Nous renvoyons ici à Jean Duvignaud, Sociologie de l'art, Paris, PUF, 1972.

47. Ibid., p.21.

48. Ibid., p.20.

49. Ibid., p.21 : «cette image, l'artiste peut la reprendre à son compte pour l'exalter dans la mesure où cette représentation de la nature est, en fait, l'exaltation d'une certaine figuration de l'homme pour un groupe et la mesure d'un dynamisme collectif».

50. Jean Duvignaud, op. cit., p. 21.

51. Roger Bastide, op. cit., p. 182.

52. Jean Duvignaud, op. cit., p. 21.

53. Voir la typologie des attitudes esthétiques que propose Duvignaud, ibid., p. 59-90.

54. Daniel Charles, Mikel Dufresne et Anne Sauriau, "Esthétique», Encyclopaedia Universalis, loc. cit., p. 721.

55. Michel Freitag, Le naufrage de I'université, Québec/Paris, Nuit Blanche/La Découverte, 1995, p. 262.

56. Michel Freitag, Architecture et société, op. cit., p. 10.

57. Pour Michel Freitag, l'immédiat, au sens d’absence de médiation symbolique ou institutionnelle, implique la cessation de l'activité véritablement humaine et subjective. II affirme conséquemment que cl'immédiat est l'abolissement de l'art, de l'expérience esthétique et de la beauté», voir "Notes pour une sociologie de l'art», op. cit., p. 35. 